\title{
Expedient Synthesis of Heterobifunctional Triarylmethane Stoppers for Macromolecular Rotaxanes
}

DOI:

10.1021/acs.joc.9b03063

\section{Document Version}

Accepted author manuscript

Link to publication record in Manchester Research Explorer

\section{Citation for published version (APA):}

Zhang, M., Shvetsova, O., \& De Bo, G. (2020). Expedient Synthesis of Heterobifunctional Triarylmethane Stoppers for Macromolecular Rotaxanes. The Journal of organic chemistry, 85(4), 2770-2774.

https://doi.org/10.1021/acs.joc.9b03063

\section{Published in:}

The Journal of organic chemistry

\section{Citing this paper}

Please note that where the full-text provided on Manchester Research Explorer is the Author Accepted Manuscript or Proof version this may differ from the final Published version. If citing, it is advised that you check and use the publisher's definitive version.

\section{General rights}

Copyright and moral rights for the publications made accessible in the Research Explorer are retained by the authors and/or other copyright owners and it is a condition of accessing publications that users recognise and abide by the legal requirements associated with these rights.

\section{Takedown policy}

If you believe that this document breaches copyright please refer to the University of Manchester's Takedown Procedures [http://man.ac.uk/04Y6Bo] or contact uml.scholarlycommunications@manchester.ac.uk providing relevant details, so we can investigate your claim.

\section{OPEN ACCESS}




\title{
Expedient Synthesis of Heterobifunctional Triarylmethane Stoppers for Macromolecular Rotaxanes
}

\author{
Min Zhang, Olga Shvetsova and Guillaume De Bo* \\ Department of Chemistry, University of Manchester, Oxford Road, Manchester, M13 9PL, United Kingdom \\ *guillaume.debo@manchester.ac.uk
}

\begin{abstract}
Increasingly complex rotaxane-based molecular devices are interfaced with polymers and surfaces but suitable bifunctional stoppering groups are lacking. Here we report a 2-step, high-yielding synthesis towards a new class of heterobifunctional triarylmethane stoppers. They possess hydroxyl and ester groups for further functionalization, as well as halogen substituents conferring a diagnostic spectroscopic signature. Their utility was demonstrated with the synthesis of a chain-centered macromolecular rotaxane. This new stopper architecture should prove useful to connect rotaxanes with polymers and surfaces, for applications in polymer mechanochemistry, single-molecule force spectroscopy, smart-materials, and molecular machines.
\end{abstract}

Heterobifunctional stoppers are particularly useful to interface rotaxane-based molecular machines and devices with polymers $^{1 \mathrm{a}-\mathrm{e}}$ and surfaces. ${ }^{2 \mathrm{a}-\mathrm{g}}$ However, the most popular are only suitable for relatively small macrocycles and the increasing complexity of rotaxane-based molecular devices is often accompanied by the use of larger, tailored-made, macrocycles. ${ }^{3 a-}$ ${ }^{\mathrm{h}}$ This need has been fulfilled with Gibson's very popular 'bulky stopper' ${ }^{4}$ and, increasingly, with commercially available alternatives such as 4 (Figure $1 b$ ), ${ }^{5 a-f}$ which is slightly smaller but has the advantage of displaying an enhanced solubility in organic solvent, and a diagnostic fingerprint in NMR and MS spectra. Homobifunctional counterparts of the 'bulky stopper' are available ${ }^{6}$ but no, easily accessible, heterobifunctional equivalent exists. Here we report the rapid synthesis of heterobifunctional triarylmethane stoppers 3 derived from 4 with diagnostic spectroscopic signatures and/or sites for further functionalization (Figure 1a). These stoppers should prove useful to connect rotaxanes with polymers and surfaces, for applications in polymer mechanochemistry, single-molecule force spectroscopy, materials chemistry, and molecular machines. This concept is demonstrated with the synthesis of a chain-centered macromolecular [2]rotaxane.

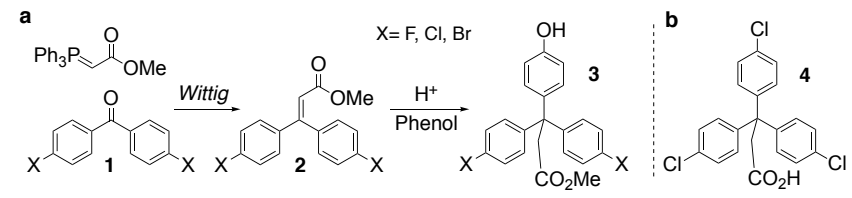

Figure 1. (a) Synthetic strategy towards heterobifunctional stopper 3. (b) Commercially available stopper 4.

Our recent investigation of the mechanochemistry of the mechanical bond, ${ }^{5 a-b, 7}$ lead us to assemble complex macromolecular [2] rotaxanes requiring a bulky heterobifunctional stopper. ${ }^{5 a}$ Inspired by Gibson's synthesis, ${ }^{4}$ which proceeds by the acid-catalyzed addition of phenol on triarylmethanol derivatives, we devised a strategy based on the addition of phenol onto conjugated ester $\mathbf{2}$ (which is formed on gram-scale from cheap commercially available diaryl ketones $\mathbf{1}$ and a Wittig reagent, Figure 1). Our initial attempt, reproducing the conditions used by Gibson, lead entirely to the formation of decarboxylated product 5a (Entry 1, Table 1). Lowering the temperature enables the formation of a small amount of the desired product (3a) but at the expense of the total conversion (Entry 2-3). As chloride ions can assist decarboxylation via a Krapcho mechanism, ${ }^{8}$ we hypothesized that acids with non-nucleophilic counterion could prevent the formation of decarboxylated product 5a. Indeed, the use of $\mathrm{HBF}_{4}$ lead to the formation of $\mathbf{3 a}$ as the major product (Entry 4). Hoping to further diminish the decarboxylation, we performed the reaction in presence of molecular sieve that could arise from the hydrolyzed ester but this proved unsuccessful (Entry 5). Using p-toluenesulfonic acid (TsOH) as a catalyst afforded 3a with moderate yield and selectivity after $16 \mathrm{~h}$ at $90^{\circ} \mathrm{C}$ (Entry 6). A slightly improved conversion was obtained with triflic acid (TfOH) in the same conditions (Entry 7). Reducing the reaction time to $1 \mathrm{~h}$ significantly improved the formation of 3a (Entry 8). Pleasingly, we found that 3a could be formed quantitively and with complete selectivity after $15 \mathrm{~min}$ at $90^{\circ} \mathrm{C}$ (Entry 9) or by maintaining the reaction mixture just above the melting point of phenol $\left(41^{\circ} \mathrm{C}\right)$ for $45 \mathrm{~min}$ (Entry 10$)$.

Table 1. Screening the reaction conditions ${ }^{a}$

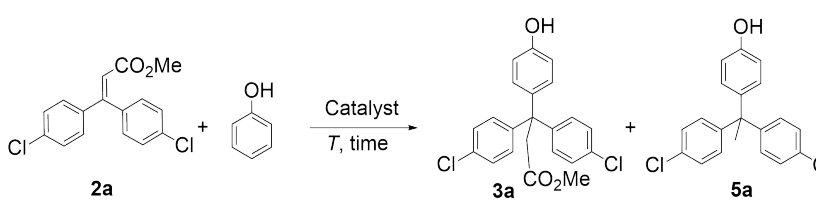

\begin{tabular}{llllll}
\hline Entry & Catalyst & $\mathrm{T}\left({ }^{\circ} \mathrm{C}\right)$ & $\begin{array}{l}\text { Time } \\
(\mathrm{h})\end{array}$ & $\begin{array}{l}\text { Yield of } \\
\mathbf{3 a}(\%)^{c}\end{array}$ & $\mathbf{3 a : 5 a}{ }^{d}$ \\
\hline 1 & $\mathrm{HCl}$ & 170 & 16 & - & $1: 100$ \\
2 & $\mathrm{HCl}$ & 90 & 16 & 7 & $1: 6$ \\
3 & $\mathrm{HCl}$ & 65 & 16 & 7 & $1: 3$ \\
4 & $\mathrm{HBF}_{4}$ & 90 & 16 & 54 & $1: 0.7$ \\
$5^{b}$ & $\mathrm{HBF}_{4}$ & 90 & 16 & 17 & $1: 1.2$ \\
6 & $\mathrm{TsOH}^{b}$ & 90 & 16 & 26 & $1: 1.9$ \\
7 & $\mathrm{TfOH}$ & 90 & 16 & 31 & $1: 2.2$ \\
8 & $\mathrm{TfOH}$ & 90 & 1 & 83 & $1: 0.2$ \\
\hline
\end{tabular}




\begin{tabular}{llllll}
\hline 9 & TfOH & 90 & 0.25 & 99 & $>100: 1$ \\
10 & TfOH & 45 & 0.75 & 99 & $>100: 1$ \\
\hline
\end{tabular}

${ }^{a}$ Reaction conditions: $2 \mathrm{a}(0.2 \mathrm{mmol}, 1.0 \mathrm{eq}$.$) , phenol (5.3 \mathrm{mmol}$, $26.5 \mathrm{eq})$, catalyst ( $0.08 \mathrm{mmol}, 0.4$ eq. $)$ under $\mathrm{N}_{2}$ atmosphere. ${ }^{b}$ molecular sieve (1.0 eq.) was added. ${ }^{c}$ Isolated yield. ${ }^{d}$ Determined by ${ }^{1} \mathrm{H}$ NMR.

The scope of the reaction was explored by reacting phenol with a range of 3,3-diarylacrylates under the optimized conditions presented in Entry 10 of Table 1. All halogen-substituted substrates showed quantitative conversion (Entry 1-3, Table 2), with no detectable amount of decarboxylated product. However, the presence of electron-donating groups on the acceptor completely suppresses its reactivity (Entry 4). Advantageously, this reaction can be easily scaled-up to gram-scale, as demonstrated by the production of $1.5 \mathrm{~g}$ of chloro derivative $3 \mathrm{a}$ in $94 \%$ yield (See SI).

\section{Table 2. Scope of the reaction ${ }^{a}$}

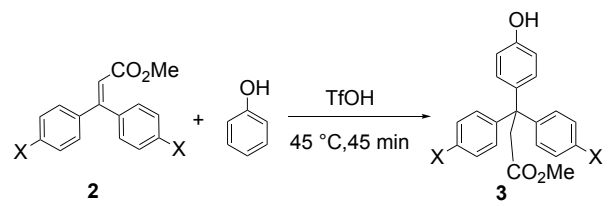

\begin{tabular}{lllll}
\hline Entry & $\mathbf{2}$ & $\mathrm{X}$ & $\mathbf{3}$ & Yield of $\mathbf{3}^{b}(\%)$ \\
\hline 1 & $\mathbf{2 a}$ & $\mathrm{Cl}$ & $\mathbf{3 a}$ & 99 \\
3 & $\mathbf{2 b}$ & $\mathrm{Br}$ & $\mathbf{3 b}$ & 96 \\
4 & $\mathbf{2 c}$ & $\mathrm{F}$ & $\mathbf{3 c}$ & 99 \\
5 & $\mathbf{2 d}$ & $\mathrm{OMe}$ & $\mathbf{3 d}$ & - \\
\hline
\end{tabular}

${ }^{a}$ Reaction conditions: 2 ( $0.2 \mathrm{mmol}, 1.0$ eq.), phenol (5.3 mmol, $26.5 \mathrm{eq})$, TsOH (0.08 mmol, 0.4 eq.) under $\mathrm{N}_{2}$ atmosphere. ${ }^{b}$ Isolated yield.

To further demonstrate the utility of this new stopper we devised the synthesis of a chain-centered rotaxane starting from ester 3c (Figure 2a). We anticipated that the para-fluoro substituents would confer a distinctive pattern in the ${ }^{1} \mathrm{H}$ and ${ }^{19} \mathrm{~F}$ NMR spectra of the various synthetic intermediates (Figure 2b-c,e). Propargylation and subsequent hydrolysis of 3c afford acid 7, which adequately possesses an acid functionality, to connect to the axle of the rotaxane, and an alkyne, to append a polymer precursor by $\mathrm{Cu}$-catalyzed azide-alkyne cycloaddition $(\mathrm{Cu}-$ AAC). Rotaxane 11 was obtained in $42 \%$ yield using Takata's method, ${ }^{9}$ by reacting activated stopper $\mathbf{8}$, diethanolamine $\mathbf{9}$, and dibenzo-24-crown-8 (DB24C8) macrocycle in presence of silver triflate. The ${ }^{1} \mathrm{H}$ NMR spectra (Figure 2e) of DB24C8 macrocycle, free-thread 10 (i.e. 11 without macrocycle) and rotaxane 11 confirms the interlocked structure. The upfield shift of axle protons $H_{\mathrm{m}}\left(\Delta \delta \mathrm{H}_{\mathrm{m}}=-0.43 \mathrm{ppm}\right)$, ammonium protons $H_{\mathrm{q}}$ $\left(\Delta \delta \mathrm{H}_{\mathrm{q}}=-0.24 \mathrm{ppm}\right)$, and macrocycle protons $H_{6}\left(\Delta \delta \mathrm{H}_{6}=-0.16\right.$ ppm), and downfield shift of axle protons $H_{\mathrm{p}}\left(\Delta \delta \mathrm{H}_{\mathrm{p}}=0.60 \mathrm{ppm}\right)$ in the rotaxane compared to the free-thread/macrocycle are indicative of the presence of the macrocycle around the ammonium station. The ${ }^{19} \mathrm{~F}$ NMR spectra (Figure $2 \mathrm{~b}$ ) clearly shows the peaks associated with the triflate anion $(-78.1 \mathrm{ppm})$, and the fluorophenyl groups (-116.3 ppm). Rotaxane 11 was then appended with polymerisation initiators (12) by $\mathrm{CuAAC}$ to reach rotaxane 13. This reaction was catalyzed by $\mathrm{Cu}(\mathrm{MeCN})_{4} \mathrm{PF}_{6}$ and ${ }^{19} \mathrm{~F}$ NMR analysis revealed the exchange of $\mathrm{TfO}^{-}$counterion for PF6 $^{-}$in the process by the presence of a doublet at $-72.6 \mathrm{ppm}$
(Figure 2c). Finally, chain-centered macromolecular rotaxane 14 was obtained by single electron transfer living radical polymerization (SET-LRP) ${ }^{10}$ of methyl acrylate initiated from both ends of the axle. GPC $\left(M_{n}=45 \mathrm{kDa}, \emptyset=1.16\right.$, Figure $\left.2 \mathrm{~d}\right)$ and ${ }^{1} \mathrm{H}$ NMR (see SI) indicate the successful incorporation of the rotaxane in the polymer.

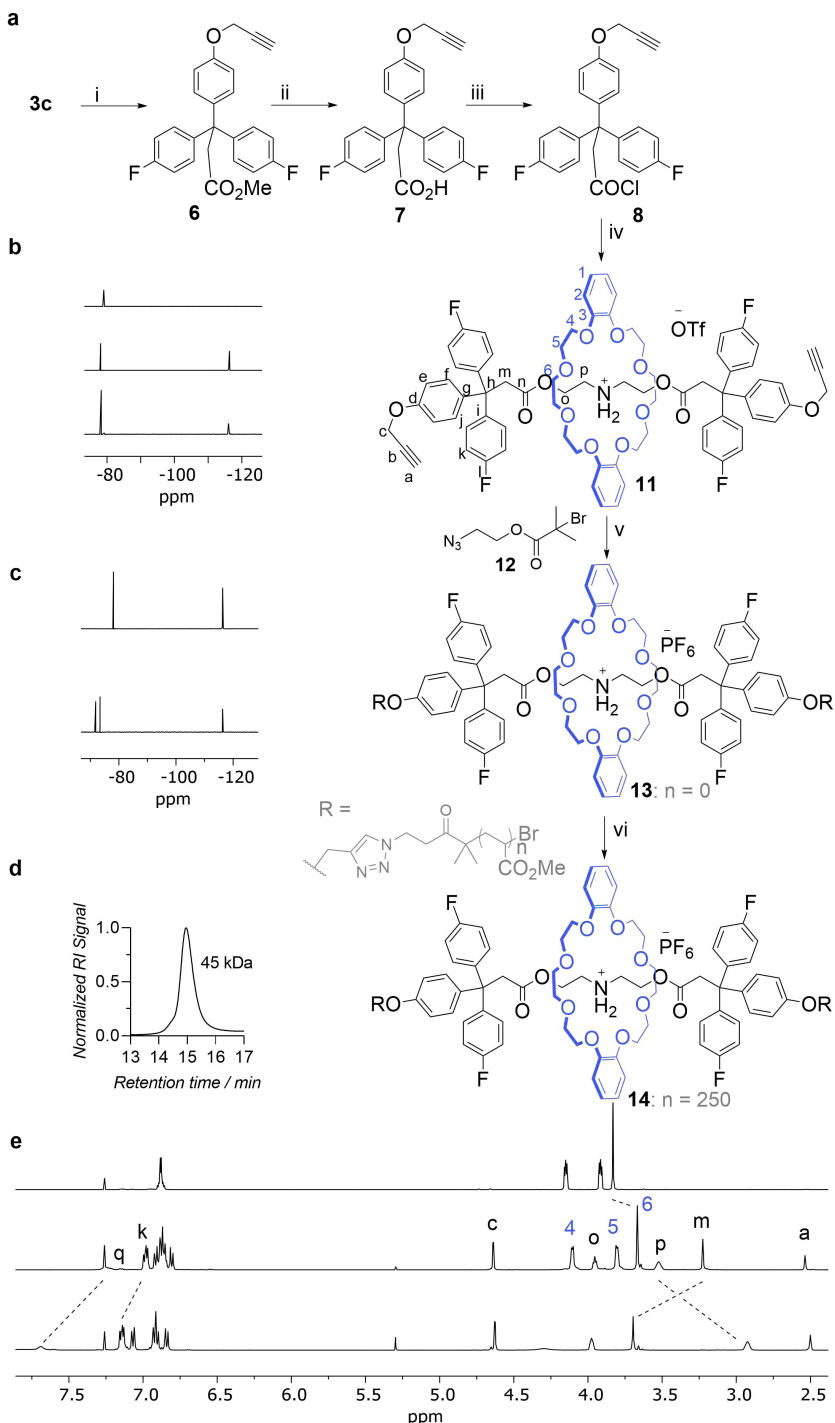

Figure 2. (a) Synthetic route towards chain-centered polymer 11. i) Propargyl bromide, $\mathrm{K}_{2} \mathrm{CO}_{3}, \mathrm{MeCN}, 70{ }^{\circ} \mathrm{C}, 12 \mathrm{~h}, 96 \%$. ii) $\mathrm{LiOH}, \mathrm{H}_{2} \mathrm{O} / \mathrm{THF}, 1 \mathrm{M} \mathrm{HCl}, 12 \mathrm{~h}$, rt, quantitative. iii) $(\mathrm{COCl})_{2}$, $\mathrm{DCM}, \mathrm{rt}, 2 \mathrm{~h}$, quantitative. iv) $\mathrm{DB} 24 \mathrm{C} 8$, diethanolamine 9 , AgOTf, 2 h, rt, 42\%. v) 12, $\mathrm{Cu}(\mathrm{MeCN})_{4} \mathrm{PF}_{6}, \mathrm{DCM} / \mathrm{MeCN}, 12$ h, rt, 74\%. vi) Methyl acrylate, $\mathrm{CuBr}_{2} / \mathrm{Me}_{6} \mathrm{TREN}$, Copper wire, DMSO, rt, 40 min. (b) ${ }^{19} \mathrm{~F}$ NMR comparison of $9 \cdot \mathrm{H}^{+} \mathrm{TfO}^{-}$(top), rotaxane 11 (middle) and free thread 10 (bottom). (c) ${ }^{19} \mathrm{~F}$ NMR comparison of rotaxane $\mathbf{1 1}$ (top) and rotaxane $\mathbf{1 3}$ (bottom). (d) GPC trace of rotaxane centered polymer 14. (e) Partial ${ }^{1} \mathrm{H}$ NMR comparison of DB24C8 (top), rotaxane 11 (middle) and free thread 10 (bottom).

In conclusion, we have developed an expedient synthesis of a new class of heterobifunctional stoppers. These stoppers can be produced on gram-scale with high yields, and their halogen substituents provide them with a diagnostic spectroscopic signature and potential sites for further functionalization. The utility of these building blocks was demonstrated with the synthesis of a chain-centered macromolecular rotaxane. We anticipate that 
this new stopper architecture should prove useful to connect rotaxanes with polymers and surfaces, for applications in polymer mechanochemistry, single-molecule force spectroscopy, materials chemistry, and molecular machines.

\section{EXPERIMENTAL SECTION}

Unless otherwise stated, all reagents and solvents were purchased from commercial suppliers and used without further purification. $\mathrm{Cu}(0)$ wire (diameter: $0.25 \mathrm{~mm}$, purity $99.9 \%$ ) was purchased from Sigma-Aldrich. $\mathrm{Me}_{6}$ TREN was purchased from Alfa Aesar. Compounds $\mathbf{2} \mathrm{a}^{5 \mathrm{a}}$ and $\mathbf{1 2}^{11}$ were prepared according to literature procedures. Gel permeation chromatography (GPC) analyses were performed in THF solution $\left(\sim 0.6 \mathrm{mg} \mathrm{mL}^{-1}\right)$ at $35^{\circ} \mathrm{C}$ using a Malvern Viscotek GPCmax VE2001 solvent/sample module with $2 \times$ PL gel $10 \mu \mathrm{m}$ mixed-B and a PL gel $500 \AA$ column, and equipped with a Viscotek VE3580 refractive index detector employing narrow polydispersity polystyrene standards (Agilent Technologies) as a calibration reference. Samples were filtered through a Whatman Puradisc $4 \mathrm{~mm}$ syringe filter with $0.45 \mu \mathrm{m}$ PTFE membrane before injection to equipment, and experiments were carried out with injection volume of $200 \mu \mathrm{L}$, flow rate of $1 \mathrm{~mL} \mathrm{~min}^{-1}$. Results were analyzed using $n$-dodecane as internal marker using Malvern OmniSEC 5.10 software. Analytical TLC was performed on precoated silica gel plates ( $0.25 \mathrm{~mm}$ thick, $60 \mathrm{~F} 254$, Merck, Germany) and observed under UV light or stained with a phosphomolybdic acid solution. Preparative TLC was performed on precoated silica gel plates: 2 mm, UNIPLATE GF, Analtech Inc., DE, USA. Flash column chromatography was performed with silica gel 60 (230-400 mesh) from Sigma-Aldrich. ${ }^{1} \mathrm{H}$ and ${ }^{13} \mathrm{C}$ NMR spectra were obtained using either a Bruker Avance III $500 \mathrm{MHz}$ Prodigy instrument or a Bruker Avance III $400 \mathrm{MHz}$ Prodigy instrument at the University of Manchester. Chemical shifts are reported in parts per million (ppm) from high to low frequency and referenced to the residual solvent resonance. Coupling constants $(J)$ are reported in Hertz $(\mathrm{Hz})$ and splitting patterns are designated as follows: $\mathrm{b}=$ broad, $\mathrm{s}=$ singlet, $\mathrm{d}=$ doublet, $\mathrm{t}=$ triplet, $\mathrm{q}=$ quartet, $\mathrm{p}=$ pentet and $\mathrm{m}=$ multiplet. Mass spectra were obtained through the Mass Spectrometry services in the Department of Chemistry at the University of Manchester.

General procedure for the synthesis of 3,3-diarylacrylates 2 A solution of 1 ( $2 \mathrm{mmol}, 1.0$ eq.) and methyl (triphenylphosphoranylidene) acetate $(2.4 \mathrm{mmol}, 1.2 \mathrm{eq}$.$) in toluene (5 \mathrm{~mL})$ was heated to reflux for $36 \mathrm{~h}$ in an oil bath. The solvent was removed and the crude product was purified by flash column chromatography $(\mathrm{PE}: \mathrm{EtOAc}=10: 1)$ to give $\mathbf{2}$.

\section{Methyl 3,3-bis(4-bromophenyl)acrylate $2 \boldsymbol{b}$}

White solid, mp 103.4-105.2 ${ }^{\circ} \mathrm{C}$; yield 74\% (586 mg); ${ }^{1} \mathrm{H}$ NMR $\left(500 \mathrm{MHz}, \mathrm{CDCl}_{3}\right) \delta=7.52(\mathrm{~d}, J=7.0 \mathrm{~Hz}, 2 \mathrm{H}), 7.46(\mathrm{~d}, J=7.0$ $\mathrm{Hz}, 2 \mathrm{H}), 7.13(\mathrm{~d}, J=7.0 \mathrm{~Hz}, 2 \mathrm{H}), 7.06(\mathrm{~d}, J=7.0 \mathrm{~Hz}, 2 \mathrm{H}), 6.35(\mathrm{~s}$, $1 \mathrm{H}), 3.64(\mathrm{~s}, 3 \mathrm{H}) ;{ }^{13} \mathrm{C}\left\{{ }^{1} \mathrm{H}\right\} \mathrm{NMR}\left(126 \mathrm{MHz}, \mathrm{CDCl}_{3}\right) \delta=166.1$, $154.8,139.4,137.2,131.9,131.5,131.0,129.9,124.4,123.0$, 117.7, 117.7, 51.6, 51.6; MS-ESI(+): $\mathrm{m} / \mathrm{z}=417.0\left(50,[\mathrm{M}+\mathrm{Na}]^{+}\right)$; $419.0\left(100,[\mathrm{M}+\mathrm{Na}]^{+}\right) ; 421.0\left(30,[\mathrm{M}+\mathrm{Na}]^{+}\right)$; HRMS (ESIFTMS) m/z: $[\mathrm{M}+\mathrm{H}]^{+}$Calcd for $\mathrm{C}_{16} \mathrm{H}_{13} \mathrm{O}_{2} \mathrm{Br}_{2}$ 394.9277; Found 394.9281 .

\section{Methyl 3,3-bis(4-fluorophenyl)acrylate 2c}

White solid, mp 46.1-48.0 ${ }^{\circ} \mathrm{C}$; yield 99\% (543 mg); ${ }^{1} \mathrm{H}$ NMR (500 $\left.\mathrm{MHz}, \mathrm{CDCl}_{3}\right) \delta=7.16\left(\mathrm{dd}, J=8.5,9.0 \mathrm{~Hz}, 4 \mathrm{H}, H_{\mathrm{b}}\right), 7.02(\mathrm{~d}, J=$ $8.5 \mathrm{~Hz}, 2 \mathrm{H}), 6.95(\mathrm{dd}, J=8.5,9.0 \mathrm{~Hz}, 4 \mathrm{H}), 6.72(\mathrm{~d}, J=8.5 \mathrm{~Hz}, 2 \mathrm{H})$, $4.92(\mathrm{~s}, 1 \mathrm{H}), 3.63(\mathrm{~s}, 2 \mathrm{H}), 3.42(\mathrm{~s}, 3 \mathrm{H}) ;{ }^{13} \mathrm{C}\left\{{ }^{1} \mathrm{H}\right\} \mathrm{NMR}(126 \mathrm{MHz}$, $\left.\mathrm{CDCl}_{3}\right) \delta=171.5,161.3(\mathrm{~d}, J=246 \mathrm{~Hz}), 154.1,142.5(\mathrm{~d}, J=3.3$ $\mathrm{Hz}), 138.5,130.7$ (d, $J=7.8 \mathrm{~Hz}), 130.3,114.9,114.8$ (d, $J=21.1$ $\mathrm{Hz}), 54.3,51.7$ (d, $J=2.0 \mathrm{~Hz}), 46.6 ;{ }^{19} \mathrm{~F}$ NMR $\left(470 \mathrm{MHz}, \mathrm{CDCl}_{3}\right)$ $\delta=-116.63 ;$ MS-ESI(-): m/z = $367.2\left(100,[\mathrm{M}-\mathrm{H}]^{-}\right)$; HRMS (ESIFTMS) m/z: [M - H] Calcd for $\mathrm{C}_{22} \mathrm{H}_{17} \mathrm{O}_{3} \mathrm{~F}_{2}$ 367.1151; Found 367.1159 .

Methyl 3,3-bis(4-methoxyphenyl)acrylate 2d

White solid, mp 35.5-37.0 ${ }^{\circ} \mathrm{C}$; yield $21 \%$ (125 mg); ${ }^{1} \mathrm{H}$ NMR (500 $\left.\mathrm{MHz} \mathrm{CDCl}_{3}\right) \delta=7.24(\mathrm{~d}, J=8.0 \mathrm{~Hz}, 2 \mathrm{H}), 7.15(\mathrm{~d}, J=8.5 \mathrm{~Hz}, 2 \mathrm{H})$, $6.91(\mathrm{~d}, J=8.6 \mathrm{~Hz}, 2 \mathrm{H}), 6.84(\mathrm{~d}, J=8.4 \mathrm{~Hz}, 2 \mathrm{H}), 6.23(\mathrm{~s}, 1 \mathrm{H}), 3.85$ $(\mathrm{s}, 3 \mathrm{H}), 3.82(\mathrm{~s}, 3 \mathrm{H}), 3.62(\mathrm{~s}, 3 \mathrm{H}) ;{ }^{13} \mathrm{C}\left\{{ }^{1} \mathrm{H}\right\}$ NMR $(126 \mathrm{MHz}$, $\left.\mathrm{CDCl}_{3}\right) \delta=166.9,160.9,159.8,157.1,134.0,131.2,131.0,130.2$, $114.4,114.5,113.8,113.4,55.5,55.5,55.4,55.3,51.3,51.3$; MS$\mathrm{ESI}(+): \mathrm{m} / \mathrm{z}=321.2\left(30,[\mathrm{M}+\mathrm{Na}]^{+}\right) ;$HRMS (ESI-FTMS) m/z: [M $+\mathrm{Na}]^{+}$Calcd for $\mathrm{C}_{18} \mathrm{H}_{18} \mathrm{O}_{4} \mathrm{Na}$ 321.1097; Found 321.1086.

\section{General procedure for the synthesis of stopper 3}

$\mathrm{TfOH}(0.12 \mathrm{mmol}, 40 \mathrm{mmol} \%)$ was added to a mixture of $2(0.3$ mmol, 1.0 eq.) and phenol $(500 \mathrm{mg})$. The mixture was heated to 45 ${ }^{\circ} \mathrm{C}$ for $45 \mathrm{~min}$ in an oil bath. The crude product was washed with water and purified by flash column chromatography (PE : EtOAc $=5: 1$ ) to yield $\mathbf{3}$ as a solid.

Methyl 3,3-bis(4-chlorophenyl)-3-(4-hydroxyphenyl)propanoate $3 a$

White solid, mp 145.3-147.2 ${ }^{\circ} \mathrm{C}$; yield 99\% (119 mg); ${ }^{1} \mathrm{H}$ NMR $\left(400 \mathrm{MHz}, \mathrm{CDCl}_{3}\right) \delta=7.23(\mathrm{~d}, J=8.4 \mathrm{~Hz}, 4 \mathrm{H}), 7.13(\mathrm{~d}, J=8.8$ $\mathrm{Hz}, 4 \mathrm{H}), 7.02(\mathrm{~d}, J=8.8 \mathrm{~Hz}, 2 \mathrm{H}), 6.73(\mathrm{~d}, J=8.8 \mathrm{~Hz}, 2 \mathrm{H}), 4.69$ (b, 1H), 3.61 (s, 2H), 3.42 (s, 3H); ${ }^{13} \mathrm{C}\left\{{ }^{1} \mathrm{H}\right\} \mathrm{NMR}\left(126 \mathrm{MHz}, \mathrm{CDCl}_{3}\right)$ $\delta=171.5,154.4,145.0,137.7,132.5,130.5,130.2,128.2,115.0$, 54.5, 51.8, 46.2; MS-ESI(-): $\mathrm{m} / \mathrm{z}=399.1\left(100,[\mathrm{M}-\mathrm{H}]^{-}\right) ; 401.1(80$, [M - H] $]^{-}$; HRMS (ESI-FTMS) m/z: [M - H] $]^{-}$Calcd for $\mathrm{C}_{22} \mathrm{H}_{17} \mathrm{O}_{3} \mathrm{Cl}_{2}$ 399.0560; Found 399.0566.

\section{Methyl 3,3-bis(4-bromophenyl)-3-(4-hydroxyphenyl)propanoate} $3 b$

White solid, mp 162.9-164.7 ${ }^{\circ} \mathrm{C}$; yield $96 \%$ (141 mg); ${ }^{1} \mathrm{H}$ NMR $\left(500 \mathrm{MHz}, \mathrm{CDCl}_{3}\right) \delta=7.38(\mathrm{~d}, J=8.5 \mathrm{~Hz}, 4 \mathrm{H}), 7.07(\mathrm{~d}, J=8.4$ $\mathrm{Hz}, 4 \mathrm{H}), 7.01(\mathrm{~d}, J=9.0 \mathrm{~Hz}, 2 \mathrm{H}), 6.72(\mathrm{~d}, J=8.5 \mathrm{~Hz}, 2 \mathrm{H}), 4.83(\mathrm{~b}$, $1 \mathrm{H}), 3.61(\mathrm{~s}, 2 \mathrm{H}), 3.42(\mathrm{~s}, 3 \mathrm{H}) ;{ }^{13} \mathrm{C}\left\{{ }^{1} \mathrm{H}\right\} \mathrm{NMR}\left(126 \mathrm{MHz}, \mathrm{CDCl}_{3}\right)$ $\delta=171.2,154.2,145.4,137.9,131.2,130.9,130.3,120.7,115.0$, 54.6, 51.7, 46.0; MS-ESI(-): $\mathrm{m} / \mathrm{z}=487.1$ (50, [M - H] $\left.{ }^{-}\right)$; HRMS (ESI-FTMS) m/z: [M - H] Calcd for $\mathrm{C}_{22} \mathrm{H}_{17} \mathrm{O}_{3} \mathrm{Br}_{2}$ 486.9550; Found 486.9545 .

Methyl 3,3-bis(4-fluorophenyl)-3-(4-hydroxyphenyl)propanoate 3c White solid, mp 95.5-97.3 ${ }^{\circ} \mathrm{C}$; yield 99\% (109 mg); ${ }^{1} \mathrm{H}$ NMR (500 $\left.\mathrm{MHz}, \mathrm{CDCl}_{3}\right) \delta=7.16(\mathrm{dd}, J=8.5,9.0 \mathrm{~Hz}, 4 \mathrm{H}), 7.02(\mathrm{~d}, J=8.5$ $\mathrm{Hz}, 2 \mathrm{H}), 6.95(\mathrm{dd}, J=8.5,9.0 \mathrm{~Hz}, 4 \mathrm{H}), 6.72(\mathrm{~d}, J=8.5 \mathrm{~Hz}, 2 \mathrm{H})$, 4.92 (s, 1H), 3.63 (s, 2H), 3.42 (s, 3H); ${ }^{13} \mathrm{C}\left\{{ }^{1} \mathrm{H}\right\}$ NMR (126 MHz, $\left.\mathrm{CDCl}_{3}\right) \delta=171.5,161.3(\mathrm{~d}, J=246 \mathrm{~Hz}), 154.2,142.5(\mathrm{~d}, J=3.3$ $\mathrm{Hz}), 138.5,130.7$ (d, $J=7.8 \mathrm{~Hz}), 130.3,114.9,114.8$ (d, $J=21.1$ $\mathrm{Hz}), 54.3,51.7(\mathrm{~d}, J=2.0 \mathrm{~Hz}), 46.6 ;{ }^{19} \mathrm{~F} \mathrm{NMR}\left(470 \mathrm{MHz}, \mathrm{CDCl}_{3}\right)$ $\delta=-116.63 ; \operatorname{MS}-\operatorname{ESI}(-): \mathrm{m} / \mathrm{z}=367.2\left(100,[\mathrm{M}-\mathrm{H}]^{-}\right)$; HRMS (ESIFTMS) m/z: [M - H] Calcd for $\mathrm{C}_{22} \mathrm{H}_{17} \mathrm{O}_{3} \mathrm{~F}_{2}$ 367.1151; Found 367.1159 .

\section{Synthesis of rotaxane 13}

Methyl 3,3-bis(4-fluorophenyl)-3-(4-(prop-2-yn-1-yloxy)phenyl)propanoate 6

To a solution of $3 \mathbf{c}(850 \mathrm{mg}, 2.3 \mathrm{mmol}, 1.0 \mathrm{eq}$.) and propargyl bromide $(550 \mathrm{mg}, 4.6 \mathrm{mmol}, 2.0$ eq.) in $\mathrm{MeCN}(5 \mathrm{~mL})$ was added $\mathrm{K}_{2} \mathrm{CO}_{3}$ (476 mg, $3.45 \mathrm{mmol}, 1.5$ eq.). The mixture was heated to $70{ }^{\circ} \mathrm{C}$ in an oil bath overnight. The crude was washed with $\mathrm{NH}_{4} \mathrm{Cl}$ (aq. $5 \mathrm{~mL})$ and extracted with DCM $(3 \times 5 \mathrm{~mL})$. The organic phase was combined and purified by flash column chromatography (PE : EtOAC $=10: 1)$ to yield 6 as a white solid (900 mg, 96\% yield), mp 39.5-41.4 ${ }^{\circ} \mathrm{C} .{ }^{1} \mathrm{H}$ NMR $\left(500 \mathrm{MHz}, \mathrm{CDCl}_{3}\right) \delta=7.16(\mathrm{dd}, J=8.5$, $9.0 \mathrm{~Hz}, 4 \mathrm{H}), 7.09$ (d, $J=8.5 \mathrm{~Hz}, 2 \mathrm{H}), 6.95(\mathrm{dd}, J=8.5,8.5 \mathrm{~Hz}, 4 \mathrm{H})$, 
$6.83(\mathrm{~d}, J=8.5 \mathrm{~Hz}, 2 \mathrm{H}), 4.66(\mathrm{~d}, J=2.0 \mathrm{~Hz}, 2 \mathrm{H}), 3.64(\mathrm{~s}, 2 \mathrm{H}), 3.41$ $(\mathrm{s}, 3 \mathrm{H}), 2.52(\mathrm{t}, J=2.0 \mathrm{~Hz}, 1 \mathrm{H}) ;{ }^{13} \mathrm{C}\left\{{ }^{1} \mathrm{H}\right\} \mathrm{NMR}\left(126 \mathrm{MHz}, \mathrm{CDCl}_{3}\right)$ $\delta=171.3,161.4(\mathrm{~d}, J=246 \mathrm{~Hz}), 156.2,142.4(\mathrm{~d}, J=3.4 \mathrm{~Hz}), 139.5$, $130.8(\mathrm{~d}, J=7.9 \mathrm{~Hz}), 130.1,114.8(\mathrm{~d}, J=21.1 \mathrm{~Hz}), 114.3,78.7$ (d, $J=2.1 \mathrm{~Hz}), 75.7(\mathrm{~d}, J=8.1 \mathrm{~Hz}), 55.9,54.3,51.7(\mathrm{~d}, J=2.1 \mathrm{~Hz})$, 46.6; ${ }^{19} \mathrm{~F}$ NMR $\left(470 \mathrm{MHz}, \mathrm{CDCl}_{3}\right) \delta=-116.59$; $\mathrm{MS}-\mathrm{ESI}(+)$ : $\mathrm{m} / \mathrm{z}=$ $429.2\left(100,[\mathrm{M}+\mathrm{Na}]^{+}\right) ; 430.2\left(20,[\mathrm{M}+\mathrm{Na}]^{+}\right)$; HRMS (ESIFTMS) $\mathrm{m} / \mathrm{z}$ : $[\mathrm{M}+\mathrm{Na}]^{+}$Calcd for $\mathrm{C}_{25} \mathrm{H}_{20} \mathrm{O}_{3} \mathrm{~F}_{2} \mathrm{Na}$ 429.1273; Found 429.1262 .

\section{3,3-bis(4-fluorophenyl)-3-(4-(prop-2-yn-1-yloxy)phenyl)propa- noic acid 7}

To a solution of 6 (812 mg, $2.0 \mathrm{mmol}, 1.0$ eq.) in $\mathrm{MeOH}(2 \mathrm{~mL})$ was added a solution of $\mathrm{LiOH}\left(384 \mathrm{mg}, 8.0 \mathrm{mmol}, 4.0\right.$ eq.) in $\mathrm{H}_{2} \mathrm{O}$ $(2 \mathrm{~mL})$. The resulting solution was stirred at room temperature overnight. The solvent was removed and acidified using $1 \mathrm{M} \mathrm{HCl}$ until $\mathrm{pH}=2$. The crude product was extracted by DCM $(3 \times 5 \mathrm{~mL})$ and concentrated. The crude product was used directly without further purification, mp 95.2-97.0 ${ }^{\circ} \mathrm{C} .{ }^{1} \mathrm{H}$ NMR $\left(500 \mathrm{MHz}, \mathrm{CDCl}_{3}\right) \delta$ $=7.12(\mathrm{dd}, J=8.5,9.0 \mathrm{~Hz}, 4 \mathrm{H}), 7.07(\mathrm{~d}, J=8.5 \mathrm{~Hz}, 2 \mathrm{H}), 6.94(\mathrm{dd}$, $J=8.5,8.5 \mathrm{~Hz}, 4 \mathrm{H}), 6.87(\mathrm{~d}, J=9.0 \mathrm{~Hz}, 2 \mathrm{H}), 4.66(\mathrm{~d}, J=2.5 \mathrm{~Hz}$, 2H), $3.63(\mathrm{~s}, 2 \mathrm{H}), 2.52(\mathrm{t}, J=2.5 \mathrm{~Hz}, 1 \mathrm{H}) ;{ }^{13} \mathrm{C}\left\{{ }^{1} \mathrm{H}\right\}$ NMR $(126$ $\left.\mathrm{MHz}, \mathrm{CDCl}_{3}\right) \delta=176.0,161.3(\mathrm{~d}, J=246 \mathrm{~Hz}), 156.2,142.1(\mathrm{~d}, J$ $=3.4 \mathrm{~Hz}), 139.3,130.7(\mathrm{~d}, J=7.8 \mathrm{~Hz}), 130.1,114.9(\mathrm{~d}, J=21.2$ $\mathrm{Hz}), 114.4,78.6(\mathrm{~d}, J=2.0 \mathrm{~Hz}), 75.8(\mathrm{~d}, J=8.1 \mathrm{~Hz}), 55.9,54.0$, 46.4; ${ }^{19} \mathrm{~F}$ NMR $\left(470 \mathrm{MHz}, \mathrm{CDCl}_{3}\right) \delta=-116.59$.

\section{3,3-bis(4-fluorophenyl)-3-(4-(prop-2-yn-1-yloxy)phenyl)propa- noyl chloride $\mathbf{8}$}

To a solution of 7 (588 mg, $1.5 \mathrm{mmol}, 1.0$ eq.) in DCM (3 mL) was added 2 drops of DMF. $(\mathrm{COCl})_{2}(0.26 \mathrm{~mL}, 3 \mathrm{mmol}, 2.0$ eq. $)$ was added dropwise. The resulting solution was stirred at room temperature for $2 \mathrm{~h}$. The solvent was removed and dried under high vacuum. The crude product was used directly without further purification

\section{Free-thread 10}

To a dry flask was added diethanolamine $9(50 \mathrm{mg}, 79 \mu \mathrm{mol}, 1.0$ eq.) and $\operatorname{AgOTf}(45 \mathrm{mg}, 158 \mu \mathrm{mol}, 2.2$ eq.). DCM $(500 \mu \mathrm{L})$ was then added. The solution was cooled down in an ice bath before the addition of $\mathbf{8}(18 \mathrm{mg}, 79 \mu \mathrm{mol}, 1.2$ eq.). The resulting solution was stirred at room temperature overnight. The crude product was purified by flash column chromatography ( $4 \% \mathrm{MeOH}$ in DCM) yielding 10 as a white solid (15 mg, 19\% yield). ${ }^{1} \mathrm{H}$ NMR $(500 \mathrm{MHz}$, $\left.\mathrm{CDCl}_{3}\right) \delta=7.76(\mathrm{bs}, 2 \mathrm{H}), 7.14(\mathrm{dd}, J=8.5,8.5 \mathrm{~Hz}, 8 \mathrm{H}), 7.07(\mathrm{~d}, J$ $=9.0 \mathrm{~Hz}, 4 \mathrm{H}), 6.92(\mathrm{dd}, J=8.5,8.5 \mathrm{~Hz}, 8 \mathrm{H}), 6.84(\mathrm{~d}, J=9.0 \mathrm{~Hz}$, $4 \mathrm{H}), 4.63(\mathrm{~d}, J=2.0 \mathrm{~Hz}, 4 \mathrm{H}), 3.99(\mathrm{t}, J=5.0 \mathrm{~Hz}, 4 \mathrm{H}), 3.70(\mathrm{~s}, 4 \mathrm{H})$, $2.92(\mathrm{t}, J=5.5 \mathrm{~Hz}, 4 \mathrm{H}), 2.50(\mathrm{t}, J=2.5 \mathrm{~Hz}, 2 \mathrm{H}) ;{ }^{13} \mathrm{C}\left\{{ }^{1} \mathrm{H}\right\} \mathrm{NMR}$ $\left(126 \mathrm{MHz}, \mathrm{CDCl}_{3}\right) \delta=171.1,161.4(\mathrm{~d}, J=246 \mathrm{~Hz}), 156.2,147.61$, $141.1(\mathrm{~d}, J=3.3 \mathrm{~Hz}), 138.9,130.7(\mathrm{~d}, J=7.6 \mathrm{~Hz}), 130.2,114.9$ (d, $J=21.3 \mathrm{~Hz}), 114.4,78.6(\mathrm{~d}, J=2.1 \mathrm{~Hz}), 75.9(\mathrm{~d}, J=9.1 \mathrm{~Hz}), 59.0$, 55.9, 54.3, 47.3, 46.1; ${ }^{19} \mathrm{~F}$ NMR (470 MHz, $\left.\mathrm{CDCl}_{3}\right) \delta=-78.28$, 116.04; MS-ESI(+): 854.5 (100, [M - OTf $\left.{ }^{+}\right) ; 855.5$ (30, [M $\mathrm{OTf}^{+}$); HRMS (ESI-FTMS) m/z: $[\mathrm{M}-\mathrm{OTf}]^{+}$Calcd for $\mathrm{C}_{52} \mathrm{H}_{44} \mathrm{NO}_{6} \mathrm{~F}_{4}$ 854.3099; Found 854.3086.

\section{Rotaxane 11}

To a dry flask was added diethanolamine $9(50 \mathrm{mg}, 79 \mu \mathrm{mol}, 1.0$ eq.), dibenzo-24-crown-8 (51 mg, $118 \mu \mathrm{mol}, 2.0$ eq.) and AgOTf (45 mg, $158 \mu \mathrm{mol}, 2.2$ eq.). DCM (100 $\mu \mathrm{L})$ was then added. The solution was stirred at room temperature for $2 \mathrm{~h}$. The solution was cooled down in an ice bath before the addition of $\mathbf{8}(18 \mathrm{mg}, 79$ $\mu \mathrm{mol}, 1.2$ eq.). The resulting solution was stirred at room temperature overnight. The crude product was purified by flash column chromatography (4\% MeOH in DCM) yielding 11 as a white solid
(48 mg, 42\% yield). ${ }^{1} \mathrm{H} \mathrm{NMR}\left(500 \mathrm{MHz}, \mathrm{CDCl}_{3}\right) \delta=6.98(\mathrm{dd}, J=$ $8.5,9.0 \mathrm{~Hz}, 8 \mathrm{H}), 6.91(\mathrm{~d}, J=8.5 \mathrm{~Hz}, 4 \mathrm{H}), 6.89-6.84(\mathrm{~m}, 16 \mathrm{H}), 6.81$ $(\mathrm{d}, J=9.0 \mathrm{~Hz}, 4 \mathrm{H}), 4.64(\mathrm{~d}, J=2.4 \mathrm{~Hz}, 4 \mathrm{H}), 4.11(\mathrm{t}, J=3.5 \mathrm{~Hz}$, $8 \mathrm{H}), 3.95$ (t, $J=4.5 \mathrm{~Hz}, 4 \mathrm{H}), 3.81(\mathrm{t}, J=3.5 \mathrm{~Hz}, 8 \mathrm{H}), 3.67(\mathrm{~s}, 8 \mathrm{H})$, $3.52(\mathrm{t}, J=4.0 \mathrm{~Hz}, 4 \mathrm{H}), 3.22(\mathrm{~s}, 4 \mathrm{H}), 2.54(\mathrm{t}, J=2.5 \mathrm{~Hz}, 2 \mathrm{H})$; ${ }^{13} \mathrm{C}\left\{{ }^{1} \mathrm{H}\right\} \mathrm{NMR}\left(126 \mathrm{MHz}, \mathrm{CDCl}_{3}\right) \delta=170.2,161.3(\mathrm{~d}, J=246 \mathrm{~Hz})$, 156.1, 147.6, $142.0(\mathrm{~d}, J=3.3 \mathrm{~Hz}), 139.0,130.6(\mathrm{~d}, J=7.6 \mathrm{~Hz})$, $130.0,122.1,114.8(\mathrm{~d}, J=21.0 \mathrm{~Hz}), 114.3,113.0,78.7(\mathrm{~d}, J=2.6$ $\mathrm{Hz}), 75.9$ (d, $J=10.3 \mathrm{~Hz}), 71.0,70.5,68.3,59.8,55.9,54.0,47.4$, $45.5 ;{ }^{19} \mathrm{~F} \mathrm{NMR}\left(470 \mathrm{MHz}, \mathrm{CDCl}_{3}\right) \delta=-78.10,-116.32$; MS-ESI $(+)$ : $1302.9\left(100,[\mathrm{M}-\mathrm{OTf}]^{+}\right) ; 1304.9\left(20,[\mathrm{M}-\mathrm{OTf}]^{+}\right)$; HRMS (ESIFTMS) m/z: [M - OTf] ${ }^{+}$Calcd for $\mathrm{C}_{76} \mathrm{H}_{76} \mathrm{NO}_{14} \mathrm{~F}_{4} 1302.5196$; Found 1302.5155 (For isotopic distribution see $\mathrm{SI}$ ).

\section{Rotaxane 13}

To a dry flask was added $\mathbf{1 1}(10 \mathrm{mg}, 6.9 \mu \mathrm{mol}, 1.0$ eq. $)$ and 12 (5.0 $\mathrm{mg}, 20.7 \mu \mathrm{mol}, 3.0$ eq.). DCM $(0.8 \mathrm{~mL})$ and $\mathrm{MeCN}(0.2 \mathrm{~mL})$ were added. The solution was degassed 4 times by freeze-thaw cycles. The mixture was then cannulated to a degassed vial containing $\mathrm{Cu}(\mathrm{MeCN})_{4} \mathrm{PF}_{6}(5.1 \mathrm{mg}, 13.8 \mu \mathrm{mol}, 2.0$ eq. $)$. The resulting solution was stirred at room temperature overnight. The crude product was purified by flash column chromatography ( $4 \% \mathrm{MeOH}$ in DCM) yielding 13 as a white solid $\left(9.8 \mathrm{mg}, 74 \%\right.$ yield). ${ }^{1} \mathrm{H}$ NMR (500 $\left.\mathrm{MHz}_{\mathrm{CDCl}}\right) \delta=7.87$ (bs, $\left.2 \mathrm{H}\right), 6.98(\mathrm{dd}, J=8.5,8.5 \mathrm{~Hz}, 8 \mathrm{H})$, 6.89-6.82 (m, 24H), $5.16(\mathrm{~s}, 4 \mathrm{H}), 4.70(\mathrm{t}, J=5.5 \mathrm{~Hz}, 4 \mathrm{H}), 4.57(\mathrm{t}$, $J=5.5 \mathrm{~Hz}, 4 \mathrm{H}), 4.10(\mathrm{t}, J=3.5 \mathrm{~Hz}, 8 \mathrm{H}), 3.93(\mathrm{t}, J=4.5 \mathrm{~Hz}, 4 \mathrm{H})$, $3.79(\mathrm{t}, J=3.5 \mathrm{~Hz}, 8 \mathrm{H}), 3.64(\mathrm{~s}, 8 \mathrm{H}), 3.50(\mathrm{t}, J=4.0 \mathrm{~Hz}, 4 \mathrm{H}), 3.19$ $(\mathrm{s}, 4 \mathrm{H}) ;{ }^{13} \mathrm{C}\left\{{ }^{1} \mathrm{H}\right\}$ NMR $\left(126 \mathrm{MHz}, \mathrm{CDCl}_{3}\right) \delta=171.2,170.2,161.3$ $(\mathrm{d}, J=246 \mathrm{~Hz}), 156.9,147.6,144.2,142.0$ (d, $J=3.4 \mathrm{~Hz}), 138.4$, $130.6(\mathrm{~d}, J=7.8 \mathrm{~Hz}), 130.0,124.3,122.2,114.8(\mathrm{~d}, J=21.0 \mathrm{~Hz})$, $114.3,113.0,70.9,70.5,68.2,64.0,61.9,59.8,55.6,54.1,50.0$, 47.5, 45.6, 30.7; ${ }^{19} \mathrm{~F}$ NMR (470 MHz, $\left.\mathrm{CDCl}_{3}\right) \delta=-72.63(\mathrm{~d}, J=$ $712 \mathrm{~Hz}),-116.32$; MS-ESI(+): 1774.5 (100, [M-PF $\left.]^{+}\right)$; HRMS (ESI-FTMS) $\mathrm{m} / \mathrm{z}: \quad\left[\mathrm{M}-\mathrm{PF}_{6}\right]^{+}$Calcd for $\mathrm{C}_{88} \mathrm{H}_{96} \mathrm{~N}_{7} \mathrm{O}_{18} \mathrm{Br}_{2} \mathrm{~F}_{4}$ 1772.5109; Found 1772.5047 (For isotopic distribution see SI).

\section{Synthesis of rotaxane 14}

Methyl acrylate was filtered through basic alumina to remove the inhibitor prior to use. A stock solution of $\mathrm{Me}_{6} \mathrm{TREN}(16 \mu \mathrm{L}, 0.060$ $\mathrm{mmol})$ and $\mathrm{CuBr}_{2}(5.6 \mathrm{mg}, 0.025 \mathrm{mmol})$ in dry DMSO $(1 \mathrm{~mL})$ was prepared. To a $5 \mathrm{~mL}$ vial was added rotaxane $13(10 \mathrm{mg}, 5.2 \mu \mathrm{mol}$, 1.0 eq.), $42 \mu \mathrm{L}$ of catalytic solution ( $\mathrm{Me}_{6} \mathrm{TREN}: 3.0 \mu \mathrm{mol}, 0.48 \mathrm{eq}$; $\mathrm{CuBr}_{2}: 1.2 \mu \mathrm{mol}, 0.2$ eq.), methyl acrylate $(0.48 \mathrm{~mL}, 5.219 \mathrm{mmol}$, 1000.0 eq.) and dry DMSO $(0.4 \mathrm{~mL})$. This solution was degassed by bubbling with $\mathrm{N}_{2}$ for $10 \mathrm{~min}$. A Cu (0) wire $(\sim 2 \mathrm{~cm}, \sim 20 \mathrm{mg}$, $0.315 \mathrm{mmol}, \sim 100.0$ eq., cleaned in $\mathrm{HCl}_{\text {conc }}$ for $10 \mathrm{~min}$ ) wrapped around a stirrer bar was added and the solution degassed for a further 2 min before being allowed to stir for $2 \mathrm{~h}$. The solution was then precipitated out in stirring methanol, recovered and dried under a high vacuum for several days yielding a white polymer (230 $\mathrm{mg}, M_{\mathrm{n}}=45 \mathrm{kDa}, \emptyset=1.16$ ). Molecular weight and polydispersity indices were recorded using an analytical GPC that had been calibrated with polystyrene standards.

\section{ASSOCIATED CONTENT}

\section{Supporting Information}

${ }^{1} \mathrm{H},{ }^{13} \mathrm{C},{ }^{19} \mathrm{~F}$ NMR spectra and rotaxanes isotopic patterns. This material is available free of charge via the Internet at http://pubs.acs.org.

\section{AUTHOR INFORMATION}

\section{Corresponding Author}

*guillaume.debo@manchester.ac.uk

\section{Author Contributions}


The manuscript was written through contributions of all authors.

\section{ACKNOWLEDGMENT}

We thank the Royal Society for a Newton International Fellowship to M.Z., a summer studentship to O.S. (funded through a Research Grant), and a University Research Fellowship to G.D.B.

\section{REFERENCES}

(1) (a) Sato, H.; Aoki, D.; Takata, T. Synthesis and Star/Linear Topology Transformation of a Mechanically Linked ABC Terpolymer. ACS Macro Lett. 2016, 699-703. (b) Daisuke, A.; Satoshi, U.; Toshikazu Takata. Star/Linear Polymer Topology Transformation Facilitated by Mechanical Linking of Polymer Chains. Angew. Chem. Int. Ed. 2015, 54, 6770-6774. (c) Ogawa, T.; Usuki, N.; Nakazono, K.; Koyama, Y.; Takata, T. Linear-Cyclic Polymer Structural Transformation and Its Reversible Control Using a Rational Rotaxane Strategy. Chem. Commun. 2015, 51, 5606-5609. (d) Ogawa, T.; Nakazono, K.; Aoki, D.; Uchida, S.; Takata, T. Effective Approach to Cyclic Polymer From Linear Polymer: Synthesis and Transformation of Macromolecular [1]Rotaxane. ACS Macro Lett. 2015, 343-347. (e) Stoll, R. S.; Friedman, D. C.; Stoddart, J. F. Mechanically Interlocked Mechanophores by Living-Radical Polymerization From Rotaxane Initiators. Org. Lett. 2011, 13, 2706-2709.

(2) (a) Naranjo, T.; Lemishko, K. M.; de Lorenzo, S.; Somoza, Á.; Ritort, F.; Pérez, E. M.; ibarra, B. Dynamics of Individual Molecular Shuttles Under Mechanical Force. Nat. Commun. 2018, 9, 4512. (b) Sluysmans, D.; Hubert, S.; Bruns, C. J.; Zhu, Z.; Stoddart, J. F.; Duwez, A.-S. Synthetic Oligorotaxanes Exert High Forces When Folding Under Mechanical Load. Nat. Nanotech. 2018, 13, 209-213. (c) Sluysmans, D.; Devaux, F.; Bruns, C. J.; Stoddart, J. F.; Duwez, A.-S. Dynamic Force Spectroscopy of Synthetic Oligorotaxane Foldamers. Proc. Natl. Acad. Sci. USA 2018, 115, 9362-9366. (d) Lussis, P.; Svaldo-Lanero, T.; Bertocco, A.; Fustin, C.-A.; Leigh, D. A.; Duwez, A.-S. A Single Synthetic Small Molecule That Generates Force Against a Load. Nat. Nanotech. 2011, 6, 553-557. (e) Coskun, A.; Wesson, P. J.; Klajn, R.; Trabolsi, A.; Fang, L.; Olson, M. A.; Dey, S. K.; Grzybowski, B. A.; Stoddart, J. F. Molecular-Mechanical Switching at the Nanoparticle-Solvent Interface: Practice and Theory. J. Am. Chem. Soc. 2010, 132, 4310-4320. (f) Brough, B.; Northrop, B. H.; Schmidt, J. J.; Tseng, H.-R.; Houk, K. N.; Stoddart, J. F.; Ho, C.-M. Evaluation of Synthetic Linear Motor-Molecule Actuation Energetics. Proc. Natl. Acad. Sci. U.S.A. 2006, 103, 8583-8588. (g) Liu, Y.; Flood, A. H.; Bonvallet, P. A.; Vignon, S. A.; Northrop, B. H.; Tseng, H.-R.; Jeppesen, J. O.; Huang, T. J.; Brough, B.; Baller, M.; et al. Linear Artificial Molecular Muscles. 2005, 127, 9745-9759.

(3) For recent examples see: (a) De Bo, G.; Gall, M. A. Y.; Kuschel, S.; De Winter, J.; Gerbaux, P.; Leigh, D. A. An Artificial Molecular Machine That Builds an Asymmetric Catalyst. Nat. Nanotech. 2018, 13,381-385. (b) Sagara, Y.; Karman, M.; Verde-Sesto, E.; Matsuo, K.; Kim, Y.; Tamaoki, N.; Weder, C. Rotaxanes as Mechanochromic Fluorescent Force Transducers in Polymers. J. Am. Chem. Soc. 2018, 140, 1584-1587. (c) Zhu, K.; Baggi, G.; Loeb, S. J. Ring-Through-Ring Molecular Shuttling in a Saturated [3]Rotaxane. Nat. Chem. 2018, 56, 1. (d) Lim, J. Y. C.; Marques, I.; Félix, V.; Beer, P. D. Enantioselective Anion Recognition by Chiral Halogen-Bonding [2]Rotaxanes. J. Am. Chem. Soc. 2017, 139, 12228-12239. (e) Kohn, D. R.; Movsisyan, L. D.; Thompson, A. L.; Anderson, H. L. Porphyrin-Polyyne [3]- and [5]Rotaxanes. Org. Lett. 2017, 19, 348-351. (f) Chiu, S.-H.; Lee, Y.J.; Liu, K.-S.; Lai, C.-C.; Liu, Y. H.; Peng, S. M.; Cheng, R. Na+ Ions Induce the Pirouetting Motion and Catalytic Activity of [2]Rotaxanes. Chem. Eur. J. 2017, 23, 9756-9760. (g) Erbas-Cakmak, S.; Fielden, S.
D. P.; Karaca, U.; Leigh, D. A.; McTernan, C. T.; Tetlow, D. J.; Wilson, M. R. Rotary and Linear Molecular Motors Driven by Pulses of a Chemical Fuel. Science 2017, 358, 340-343. (h) Mochizuki, Y.; Ikeyatsu, K.; Mutoh, Y.; Hosoya, S.; Saito, S. Synthesis of Mechanically Planar Chiral Rac-[2]Rotaxanes by Partitioning of an Achiral [2]Rotaxane: Stereoinversion Induced by Shuttling. Org. Lett. 2017, $19,4347-4350$.

(4) Gibson, H. W.; Lee, S. H.; Engen, P. T.; Lecavalier, P.; Sze, J.; Shen, Y. X.; Bheda, M. New Triarylmethyl Derivatives: "Blocking Groups" for Rotaxanes and Polyrotaxanes. J. Org. Chem. 1993, 58, $3748-3756$.

(5) (a) Zhang, M.; De Bo, G. Mechanical Susceptibility of a Rotaxane. J. Am. Chem. Soc. 2019, 141, 15879-15883. (b) Zhang, M.; De Bo, G. Impact of a Mechanical Bond on the Activation of a Mechanophore. J. Am. Chem. Soc. 2018, 140, 12724-12727. (c) Fielden, S. D. P.; Leigh, D. A.; McTernan, C. T.; Pérez-Saavedra, B.; Vitorica Yrezabal, I. J. J. Am. Chem. Soc. 2018, 140, 6049-6052. (d) De Bo, G.; Dolphijn, G.; McTernan, C. T.; Leigh, D. A. [2]Rotaxane Formation by Transition State Stabilization. J. Am. Chem. Soc. 2017, 139, 84558457. (e) Fernandes, A.; Viterisi, A.; Aucagne, V.; Leigh, D. A.; Papot, S. Second Generation Specific-Enzyme-Activated Rotaxane Propeptides. Chem. Commun. 2012, 48, 2083. (f) Hannam, J. S.; Kidd, T. J.; Leigh, D. A.; Wilson, A. J. "Magic Rod" Rotaxanes: the Hydrogen Bond-Directed Synthesis of Molecular Shuttles Under Thermodynamic Control. Org. Lett. 2003, 5, 1907-1910.

(6) Gong, C.; Gibson, H. W. Synthesis and Characterization of a Polyester/Crown Ether Rotaxane Derived From a Difunctional Blocking Group. Macromolecules 1996, 29, 7029-7033.

(7) De Bo, G. Mechanochemistry of the Mechanical Bond. Chem. Sci. 2018, 9, 15-21.

(8) Krapcho, A. P.; Weimaster, J. F.; Eldridge, J. M.; Jahngen, E. G. E., Jr; Lovey, A. J.; Stephens, W. P. Synthetic Applications and Mechanism Studies of the Decarbalkoxylations of Geminal Diesters and Related Systems Effected in Dimethyl Sulfoxide by Water and/or by Water with Added Salts. J. Org. Chem. 2002, 43, 138-147.

(9) Kihara, N.; Shin, J.-I.; Ohga, Y.; Takata, T. Direct Preparation of Rotaxane From Aminoalcohol: Selective O-Acylation of Aminoalcohol in the Presence of Trifluoromethanesulfonic Acid and Crown Ether. Chem. Lett. 2001, 6, 592-593.

(10) Anastasaki, A.; Nikolaou, V.; Nurumbetov, G.; Wilson, P.; Kempe, K.; Quinn, J. F.; Davis, T. P.; Whittaker, M. R.; Haddleton, D. M. $\mathrm{Cu}(0)$-Mediated Living Radical Polymerization: a Versatile Tool for Materials Synthesis. Chem. Rev. 2016, 116, 835-877.

(11) Nantalaksakul, A.; Mueller, A.; Klaikherd, A.; Bardeen, C. J.; Thayumanavan, S. Dendritic and Linear Macromolecular Architectures for Photovoltaics: A Photoinduced Charge Transfer Investigation. $J$. Am. Chem. Soc. 2009, 131, 2727-2738. 
Insert Table of Contents artwork here

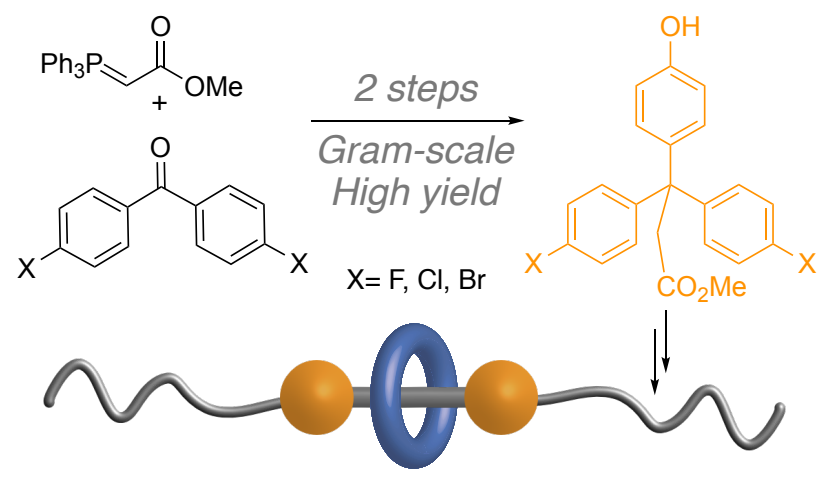

